\title{
Vers un renouveau du virus de la vaccine
}

\begin{abstract}
Premier vaccin connu, le virus de la vaccine acquiert une toute nouvelle actualité grâce à son utilisation comme vecteur de fragments exogènes d'ADN codant pour des protéines antigéniques d'intérêt vaccinal.
\end{abstract}

\section{Georges Beaud \\ Maître de Recherche au CNRS}

\section{REFEERENCES}

I. Belle Isle H, Venkatesan S, Moss B. Cell-free translation of early and late mRNAs sclected by hybridization to cloned DNA fragments derived from the left 14 million to 72 million daltons of the vaccinia virus genome. Virology $198 \mathrm{I}$; I I 2 : 306-17.

2. Mackett M, Archard L C. Conservation and variation in orthopoxvirus genome structure. $\mathcal{J}$ Gen Virol 1979; 45: 683-701.

3. Cochran MA, Puckett C, Moss B. In vitro mutagenesis of the promoter region for a vaccinia virus gene: evidence for tandem early and late regulatory signals. 7 Virol $1985 ; 54: 30-7$.

4. Berthollet C, Drillien R, Wittek R. One hundred base pairs of $5^{\prime}$ flanking sequence of vaccinia virus late gene are sufficient to temporally regulate late transcription. Proc Natl Acad Sci USA 1985; 82: 2096-100.

5. Plucienniczak A, Schroeder E, Zettlmeissl G, Streeck RE. Nucleotide sequence of a cluster of early and late genes in a conserved segment of the vaccinia virus genome. Nucleic Acids Res 1985; 13: 985-98.

\section{ADRESSE}

G. Beaud : Institut J. Monod, Tour 43, 2, place Jussieu, 7525I Paris Cedex 05. e virus de la vaccine a été employé, avec succès, comme vaccin pour éradiquer la variole et avait, par conséquent, perdu son intérêt médical. Récemment, il a été montré que ce virus pouvait être réutilisé chez l'homme (ou dans le domaine vétérinaire) pour vacciner contre d'autres maladies, puisqu'il est possible de construire des recombinants vaccine exprimant des antigènes exogènes en culture de cellules et chez l'animal. Il s'agit d'un nouveau prototype de vaccins vivants car, après inoculation à l'homme (ou l'animal), ce recombinant vaccine exprimerait suffisamment d'antigènes au cours de son développement (heureusement limité) dans l'organisme pour déclencher la formation d'anticorps protecteurs. Cette possibilité a aussi stimulé les recherches fondamentales sur l'expression des gènes du virus vaccinal. L'originalité de ce virus est que les systèmes de transcription et de réplication de l'ADN viral sont codés par le virus car les gènes vaccine sont exprimés dans le cytoplasme, alors que les autres virus à ADN utilisent les systèmes enzymatiques présents dans le noyau cellulaire.

Le virus de la vaccine (poxvirus) est un virus à $\mathrm{ADN}$ (double brin, I90 kb) de structure complexe (forme en brique de $0,2 \times 0,3 \mu \mathrm{m})$ et dont l'expression des gènes s'effectue dans le cytoplasme (figure I). Il appartient au genre des orthopox qui comprend notamment la variole, le cowpox, le rabbitpox et le monkeypox.

Des infections synchrones peuvent être effectuées avec la plupart des lignées cellulaires établies pour étudier le cycle de développement. Après l'adsorption (il n'y a pas de récepteurs membranaires spécifiques connus), le génome viral est injecté dans le cytoplasme sous la forme d'une sous-particule virale (nucléoïde) qui contient le système de transcription précoce. Les ARNm précoces sont excrétés des nucléoïdes, puis traduits en protéines précoces qui permettent la décapsidation plus complète de l'ADN parental et ensuite sa réplication. Les $A R N m$ du type tardif sont transcrits à partir de l'ADN viral dans des sites localisés du cytoplasme (virosomes). La plupart des protéines de structure sont alors synthétisées puis assemblées dans le cytoplasme. La membrane virale est également synthétisée dans les virosomes, où l'on observe ensuite la formation de virus infectieux intracellulaires. En cultures de cellules, la majorité des virions reste associée aux cellules (qui ne sont pas lysées) mais des virions peuvent être excrétés (en proportion très variable selon les souches) après un transit par le système de Golgi. Ces virions extracellulaires possèdent une dou- 

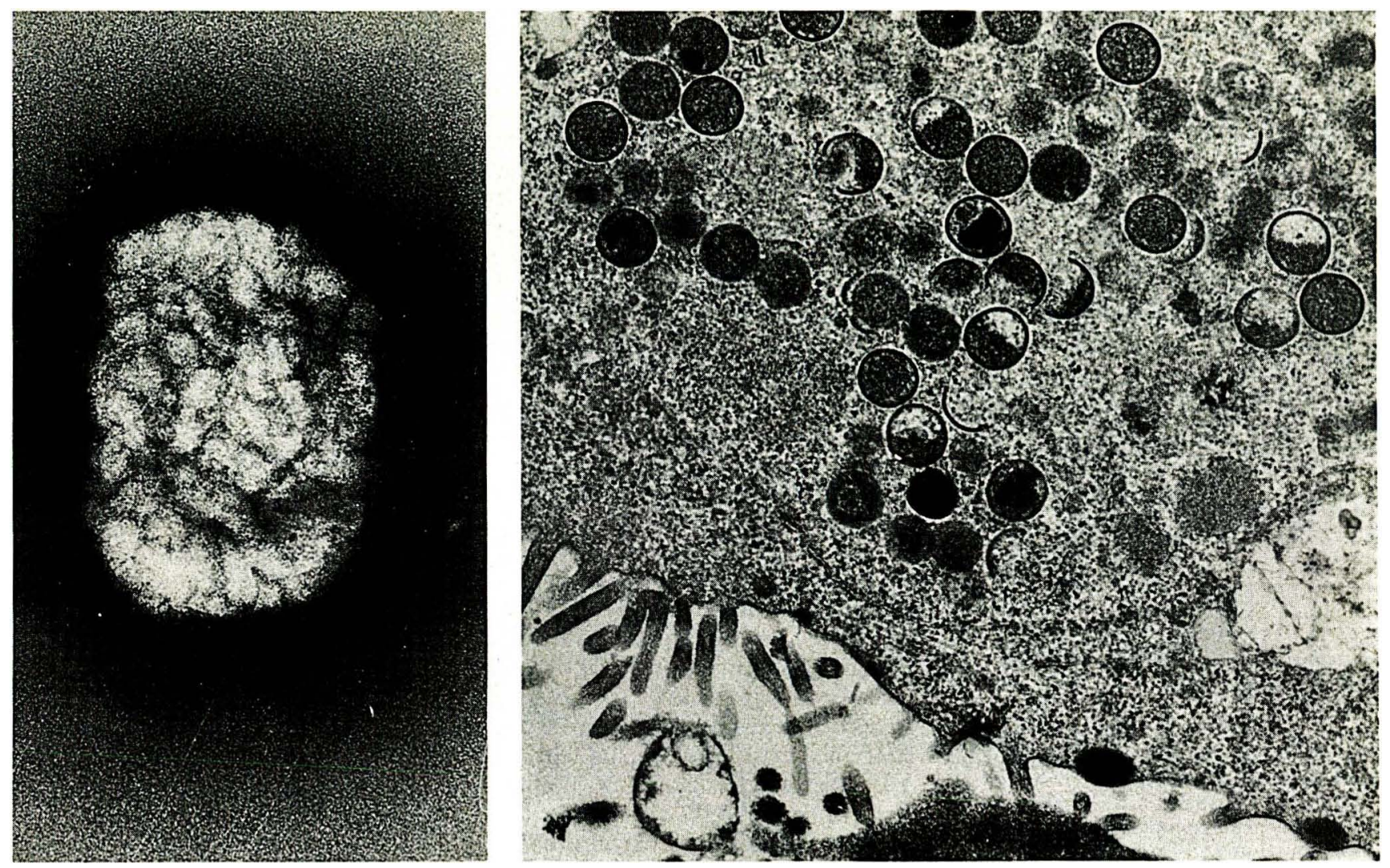

Figure I. Gauche : virus de la vaccine observé au microscope électronique après coloration négative (grandissement : $167000 \times$ ). Droite : coupe de cellules $\mathrm{Cl}$ ID infectées 24 heures par le virus vaccinal (grandissement : $23200 \times$ ). On notera la présence dans le cytoplasme de virions en cours d'assemblage à divers stades du développement et notamment l'existence de membranes virales non assemblées. La membrane plasmique est en bas, avec deux virions mûrs. (Clichés réalisés dans le Laboratoire de E. L. Benedetti, Institut 7. Monod).

ble membrane et la membrane externe est alors d'origine cellulaire. En général, plus de 10000 particules virales sont produites par cellule.

\section{Expression cytoplasmique}

Des transcriptions sélectives déclenchent la synthèse des protéines précoces, puis celle des protéines tardives. Il existe également des régulations au niveau de la traduction des ARNm, puisqu'à l'étape précoce, les ARNm cellulaires ne sont plus traduits et qu'il en est de même pour la traduction, à l'étape tardive, de la plupart des ARNm précoces synthétisés auparavant. Environ 75 protéines précoces et 40 protéines tardives ont été cartographiées sur le génome [I]. Les gènes tardifs sont plutôt rassemblés dans le centre du génome, qui est également une région bien conservée dans l'ensemble des poxvirus [2].

On peut penser que chaque gène constitue une unité indépendante de transcription. En effet jusqu'à présent, les sites de tranṡcription d'une trentaine d'ARNm précoces ont été cartographiés (méthode à la nucléase $S_{I}$, ainsi que ceux de 7 ARNm tardifs (figure 2, voir p. 97). Dans chaque cas, l'initiation de la transcription est relativement précise, bien que des origines multiples décalées de quelques bases soient parfois observees. Les séquences ADN de contrôle précèdent la séquence transcrite et sont courtes (environ 30 bases) pour les trois promoteurs (précoces et tardifs) analysés par mutagenèse in vitro $[3,4]$. Rappelons que les signaux de contrôle de la polymérase II (cellulaire) ne sont pas reconnus par le systeme de transcription vaccinal et qu'il n'y a pas d'épissage des ARN, la transcription se produisant dans le cytoplasme.

Bien que la longueur de l'ADN génomique soit de $190 \mathrm{~kb}$, l'information génétique semble très compacte, avec des séquences intergéniques très courtes, comme le révèle la cartographie des $A R N m$ effectuée jusqu'à présent (figure 2). De plus, une région de l'ADN génomique de $7,5 \mathrm{~kb}$ a été récemment séquencée [5] et, sur onze cadres de lecture possibles, trois paires adjacentes sont chevauchantes. Enfin, la séquence ARN en amont du cadre de lecture (leader) de plusieurs ARNm vaccine est réduite à quelques bases. On peut donc prévoir que les régions de con- 


\section{REFERENCES}

6. Golini F, Kates JR. A soluble transcription system derived from purified vaccinia virions. 7 . Virol 1985; 53: 205-13.

7. Puckett C, Moss B. Selective transcription of vaccinia virus genes in template dependent soluble extracts of infected cells. Cell 1983; 35: 441-8.

8. Foglesong PD. In vitro transcription of a cloned vaccinia virus gene by a soluble extract prepared from vaccinia virus-infected Hela cells. 7 . Virol 1985; 53: 822-6.

9. Vassef A, Mars M, Dru A, Plucienniczak A, Streeck RE, Beaud G. Isolation of cis-acting vaccinia virus DNA fragments promoting the expression of herpes simplex virus thymidine kinase by recombinant viruses. 7. Virol 1985; 55: 163-72.

10. Drillien R, Spehner D. Physical mapping of vaccinia virus temperature-sensitive mutations. Virology 1983; 1 31: 385-93

II. Condit RC, Motyczka A, Spizz G. Isolation, characterization and physical mapping of temperature-sensitive mutants of vaccinia virus. Virology 1983; 128: 429-43

12. Ensinger MJ, Rovinsky M. Marker rescue of temperature-sensitive mutations of vaccinia virus WR: correlation of genetic and physical maps. 7 Virol 1983; 48: 419-28.

13. Sam CK, Dumbell KR. Expression of poxvirus DNA in coinfected cells and marker rescue of thermosensitive mutants by subgenomic fragments of DNA. Annales de Virologie (Institut Pasteur) 1981; 132E: 135-50.

14. Gillard S, Spehner D, Drillien R. Mapping of a vaccinia host range sequence by insertion onto the viral thymidine kinase gene. 7 . Virol 1985; 53: 316-18.

15. Stroobant P, Rice AP, Gullick WY, Cheng DY, Kerr IM, Waterfield MD. Purification and characterization of vaccinia virus growth factor. Cell 1985; 42: 383-93.

16. Twardzik DR, Brown JP, Ranchalis JE, Todaro GJ, Moss B. Vaccinia virus-infected cells release a novel peptide functionally related to transforming and epidermal growth factors. Proc Natl Acad Sci USA 1985; 82: 5300-4.

17. Chen HR, Barker WC. Similarity of vaccinia 28K, v-erb-B and EGF receptors. Nature 1985 ; 316: $219-20$. trôle de certains ARNm pourront être transcrites (ou même traduites) dans un gène adjacent!

La terminaison de la transcription des ARNm précoces se produit dans des régions définies sur quelques dizaines de nucléotides. Les quelques cartographies des extrémités $3^{\prime}$ des ARNm précoces effectuées jusqu'à présent indiquent l'existence de terminateurs faibles (où la synthèse de l'ARN ne s'arrête pas toujours) et de terminateurs forts, ce qui suggère l'existence de deux mécanismes possibles pour la terminaison. Bien que la question ne soit pas totalement résolue, il ne semble pas que les ARNm précoces ( $\mathrm{I} \mathrm{kb}$ en moyenne) dérivent d'un précurseur par clivage enzymatique. Par contre, la terminaison des ARN tardifs est pratiquement aléatoire, puisqu'elle se produit de façon continue sur plusieurs milliers de bases! Il en résulte que la taille d'un ARNm tardif peut varier de I à $3 \mathrm{~kb}$. Cette hétérogénéité $3^{\prime}$ n'empêche pas ces ARN d'être très bien traduits, puisque l'extrémité $5^{\prime}$, elle, est fixée bien précisément.
Il est probable que, dans les prochaines années, les systèmes de transcription précoces et tardifs du virus vaccinal seront connus au niveau moléculaire. En effet, l'ARN polymérase associée aux virions a déjà été solubilisée des particules virales et purifiée partiellement (elle comporte de nombreuses sous-unités), mais l'existence d'un facteur soluble assurant l'initiation correcte de la transcription précoce n'a été suggéré que très récemment [6]. On dispose également d'extraits de cellules infectées par le virus de la vaccine, qui permettent d'initier correctement la transcription des gènes tardifs $[7,8]$. Par ailleurs, la plupart des sous-unités de l'ARN polymérase seront prochainement cartographiées sur le génome et on peut penser que la structure des signaux ADN de contrôle précoces et tardifs sera assez rapidement résolue, d'autant plus que les plus efficaces de ces signaux sont d'intérêt pratique pour la mise au point de vecteurs vaccine. Notre laboratoire a déjà isolé une dizaine de ces fragments de l'ADN du virus vaccinal [9].

\section{- GLOSS AIRE*}

ARNm précoces : ARN messagers viraux transcrits des virus infectants qui sont injectés dans le cyctoplasme.

ARNm tardifs : ARN messagers viraux transcrits de l'ADN viral répliqué dans le cytoplasme.

nucléoïdes : particules virales dont la membrane a été éliminée et constituées par l'ADN associé à une douzaine d'activités enzymatiques.

transcription : copie en $A R N$ (en général $A R N$ messager) d'un des deux brins de l'ADN. transfection : introduction de $\lceil A D N$ dans des cellules (infectées) en vue de leur intégration dans I'ADN génomique du virus de la vaccine (pour les travaux mentionnés dans cet article).

traduction : synthèse d'une protéine selon la séquence des nucléotides d'un ARN messager.

virus $\boldsymbol{R} \boldsymbol{P}$ : rabbit poxvirus (différent des virus de la myxomatose et du virus du fibrome de Shope, qui sont également des poxvirus).

virus défectif : mutant ne pouvant pas se répliquer en l'absence de co-infection par un virus apportant la fonction défectize. 


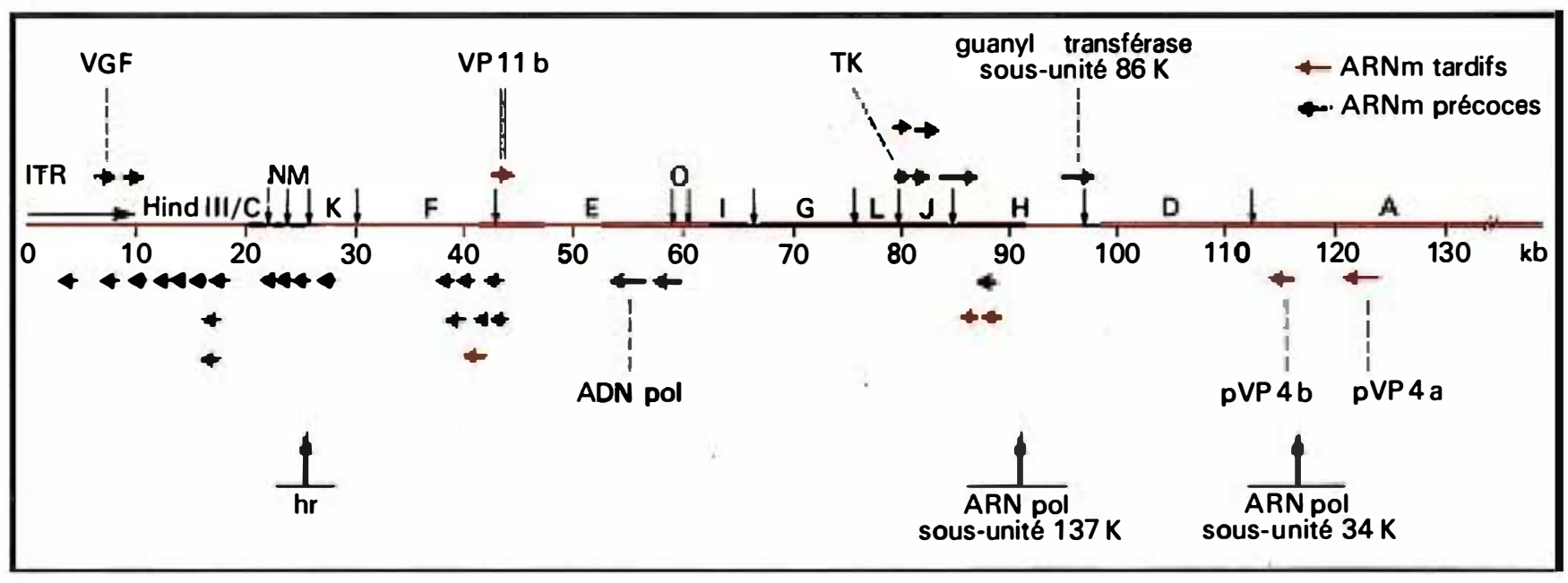

Figure 2. Cartographie des $A R N m$ du virus de la vaccine (juillet 1985). Les $130 \mathrm{~Kb}$ de la partie gauche de l'ADN génomique sont représentés avec les sites $H$ ind III (les fragments correspondants sont désignés $A-O)$ ). ITR : Inversion terminale répétée présente à chaque extrémité de l'ADN; VGF : facteur de croissance vaccinal [15, 16]; hr : gène de permissivité pour la croissance du virus de la vaccine dans les cellules humaines [14]; $A R N$ pol : deux des sous-unités de l'ARN polymérase codée par le virus vaccinal; TK: Thymidine kinase; VPIIb, pVP 4 , pVP4a: protéines de structure du virus. (Compilation des résultats publiés par diffërents laboratoires).

Dès i966, l'étude de mutants isolés du virus RP (rabbit poxvirus) avait montré qu'une analyse génétique des poxvirus était possible, car des recombinaisons génétiques très fréquentes se produisaient au cours de la réplication de l'ADN viral.

\section{Génétique du virus vaccinal}

Plus récemment, quatre collections de mutants thermosensibles (qui ne se multiplient qu'à des températures supérieures à $30^{\circ} \mathrm{C}$ ) ont été isolées. Ces mutants $t s$ se répartissent en une trentaine de groupes de complémentation et des cartes génétiques ont été établies. Les cartes physiques ont été obtenues ensuite [10, II, I2], dès que des expériences de transfection d'ADN vaccine ont été décrites [13]. L'ADN génomique n'est pas infectieux, mais il a été montré qu'un fragment d'ADN génomique (du type sauvage) transfecté par des techniques classiques dans les cellules préalablement infectées par le virus mutant, se recombine à l'ADN génomique du mutant, ce qui aboutit à la formation de virions du type sauvage. Cette technique classique du sauvetage du marqueur a permis d'établir les cartes physiques des mutants $t s$ : tous ces mutants sont situés dans la région centrale du génome et sur une centaine de $\mathrm{kb}$ seulement. Ce résultat indique qu'environ la moitié du génome concerne des gènes assurant la reproduction du virus, alors que le reste de l'ADN coderait pour des fonctions non indispensables à la reproduction des virions en culture de cellules. Cette interprétation est confirmée par l'isolement de mutants non défectifs de délétions de $10 \mathrm{~kb}$ dans la région gauche, alors que la région délétée contient une dizaine de gènes qui sont exprimés en culture de cellules. De plus, un mutant de restriction d'hôte (virus défectif pour les cellules humaines) ayant une délétion de $17 \mathrm{~kb}$ située dans la région gauche de l'ADN a été isolée; le gène correspondant a été localisé récemment [14]. Ces résultats suggèrent qu'une partie importante du génome du virus de la vaccine concerne des fonctions impliquées uniquement dans la propagation du virus dans l'organisme et donc des interactions virus-cellule dispensables selon le type de cellules infectées. La connaissance de ces fonctions serait très importante pour comprendre, au niveau moléculaire, comment un virus est pathogène. En effet, la variole et l'ectromélie sont très pathogènes, alors que la vaccine l'est très peu. On peut penser que certains gènes viraux responsables de la pathogéni- cité pourraient être similaires à des gènes cellulaires exprimés normalement à certaines étapes de la différenciation. Il n'est donc peut-être pas fortuit de trouver des analogies de séquences de certaines protéines vaccine avec des facteurs de croissance cellulaires [15, 16, 17].

\section{Un nouveau type de vaccins?}

L'originalité des recombinants vaccine comme vaccin réside dans l'inoculation d'un recombinant vaccine vivant qui exprime un antigène exogène approprié au cours de la réplication (limitée) de ce recombinant. Cet antigène doit être produit en quantité suffisante pour déclencher la réponse immunitaire. Des prototypes remplissant ces conditions chez l'animal ont déjà été obtenus. Regardons comment ils sont construits.

Nous avons vu que les recombinaisons de l'ADN vaccine répliqué dans le cytoplasme sont très fréquentes. Cela permet d'intégrer de l'ADN étranger dans le virus vaccinal dans la mesure où ces recombinaisons in vivo se produisent même si le fragment d'ADN transfecté contient une séquence d'ADN non vaccine, car une région d'homologie vaccine est présente de part et d'autre de la séquence non 


\section{REFERENCES}

18. Panicali D, Davis SW, Weinberg RL, Paoletti E. Construction of live vaccines by using genetically engineered poxviruses: biological activity of recombinant vaccinia virus expressing influenza virus hemagglutinin. Proc Natl Acad Sci USA 1983; 80: $5364-8$.

19. Smith GL, Mackett M, Moss B. Infectious vaccinia virus recombinants that express hepatitis B virus surface antigen. Nature 1983; 302: 490-5.

20. Smith GL, Moss B. Infectious poxvirus vectors have capacity for at least 25,000 base pairs of foreign DNA. Gene 1983; 25 : $21-8$.

21. Paoletti E, Lipinskas BR, Samsonoff C, Merger $S$, Panicali $D$. Construction of live vaccines using genetically engineered poxviruses: biological activity of vaccinia virus recombinants expressing the hepatitis $B$ virus surface antigen and the herpes simplex virus glycoprotein D. Proc Natl Acad Sci USA 1984; 81: 193-7.

22. Smith GL, Murphy BR, Moss B. Construction and characterization of an infectious vaccinia virus recombinant that expresses the influenza hemagglutinin gene and induces resistance to influenza virus infection in hamsters. Proc Natl Acad Sci USA 1983; 80: 71 55-9.

23. Smith GL, Godson GN, Nussenzweig V, Nussenzweig RS, Barnwell J, Moss B. Plasmodium knowlesi sporozoite antigen: expression by infectious recombinant vaccinia virus. Science 1984; 224: 397-9.

24. Kieny MP, Lathe $R$, Drillien $R$, et al., Expression of rabies virus glycoprotein from a recombinant vaccinia virus. Nature 1984; 312: 163-6.

25. Wiktor TJ, McFarlan RI, Reagan KJ, et al. Protection from rabies by a vaccinia virus recombinant containing the rabies virus glycoprotein gene. Proc Natl Acad Sci USA 1984; 81: 7194-8.

26. Moss B, Smith GL, Gerin JL, Purcell RH. Live recombinant vaccinia virus protects chimpanzees against Hepatitis B. Nature 1984; 311 : 67-9.

REVUES GENERALES SUR LE VIRUS VACCINAL :

Dales S, Pogo BGT Biology of poxvirus. In : Kingsbury DW, Zur Hausen $\mathrm{H}$, eds. Virology Monographs, Vol. 18. Springer-Verlag, 1981.

Moss B. Replication of poxviruses. In : Fields BN, ed. Virology. New York: Raven Press, 1985: 685-703.

Wittek, R. Organization and expression of the poxvirus genome. Experientia 1982; 38: 285-96. vaccine. Pour obtenir un virus non défectif, il suffit de réaliser ces insertions dans la région de l'ADN non essentielle gauche [ 18 ] ou dans le gène codant pour la thymidine kinase vaccinale situé au centre du génome [I9]. Ces expériences d'insertion d'ADN exogène sont relativement faciles à effectuer en employant les techniques classiques du génie génétique, et jusqu'à $25 \mathrm{~kb}$ d'ADN exogène ont pu être ainsi ajoutés à l'ADN vaccinal [20]. Pour assurer l'expression de séquences codantes exogènes au virus vaccinal, nous avons vu qu'il était nécessaire de placer, en amont, un promoteur du type vaccine. Jusqu'à présent, un promoteur endogène situé dans la région gauche de l'ADN viral a été employé, ainsi qu'un fragment de 275 paires de bases contenant deux promoteurs précoce et tardif, placés en tandem. Ces deux signaux ont permis d'exprimer en culture de cellules des séquences d'ADN d'intérêt médical : antigène de surface du virus de l'hépatite B [ 19 , 2I], glycoprotéine D du virus herpétique [2I], hémagglutinine du virus grippal $[18,22]$, antigène de plasmodium [23] et glycoprotéine du virus rabique [24, 25]. Dans tous les cas, il s'agit de protéines à localisation membranaire (ou excrétées des cellules), car des propriétés antigéniques sont recherchées.

L'utilisation de recombinants du virus vaccinal comme vaccin vivant pour protéger contre d'autres maladies infectieuses a été proposée par E. Paoletti et indépendamment par B. Moss, qui ont montré que l'inoculation à différents animaux (lapin, souris, hamster) de ces recombinants vaccine provoque l'apparition d'anticorps circulants. De plus, ces animaux vaccinés par un recombinant vaccine sont bien protégés contre une infection ultérieure par l'agent infectieux correspondant. Dans le cas de l'inoculation d'un recombinant hépatite $B /$ vaccine à trois chimpanzés (dont un contrôle), les deux animaux vaccinés ont résisté à l'inoculation ultérieure de virus de l'hépatite $B$ [26]. Ces expériences suggèrent qu'il devrait être possible d'utiliser les recombinants vaccine chez l'homme, et sans doute plus rapidement, dans le domaine vétérinaire

\section{Summary}

Vaccinia virus is a poxvirus containing a I90 kb doublestranded DNA which replicates in the cytoplasm. Presently, 3I early and 7 late $\mathrm{mRNAs}$ have been precisely mapped on the genome. The early and late promoters are specific to vaccinia and seem to correspond to relatively short DNA sequences which are present upstream the corresponding coding sequence. Genetic studies strongly suggest that many viral genes are not required for virus growth in cell cultures but are probably important for an efficient infection in the organism. Recently, vaccinia virus has been developed as a vector and several foreign antigens have been expressed, among them human hepatitis B surface antigen and rabies glycoprotein. The inoculation of vaccinia recombinants to laboratory animals induces a successful vaccination against the corresponding diseases. Therefore, vaccinia recombinants may be used as live vaccine in the medical and veterinary domains.

\section{TIRES A PART}

G. Beaud : Institut J. Monod, Tour 43, 2, place Jussieu, 7525I Paris Cedex o5.

$\mathrm{m} / \mathrm{s} n^{\circ} 2$ vol. 2 fëvrier 86 